\title{
Anisotropic Reaction Kinetics of Oxygen With Pyrolytic Graphite
}

\author{
William S. Horton \\ Institute for Materials Research, National Bureau of Standards, \\ Washington, D.C. 20234
}

(December 15, 1969)

\begin{abstract}
Because of the possible use of pyrolytic graphite in aerospace vehicles its reaction with oxygencontaining gases is of interest, particularly with respect to chemical anisotropy. Although several authors have found different degrees of reactivity of graphite in different directions they disagree regarding the existence of a temperature coefficient. This disagreement, however, is probably due to investigators studying single crystals in some cases and pyrolytic graphite in others and in some cases different oxidizers. New data for pyrolytic graphite are presented which were obtained by oxidizing in each run several pieces of differing geometry. These together with earlier data show that the rate ratio for the two major directions is temperature dependent with an activation energy difference of about 19 kilojoules (4500 thermochemical calories) per gram atom of carbon reacted. It is shown that this difference in rates probably arises because the relative number of sites available in these directions is sufficiently different to cause a shift in which reaction step is rate-controlling. On the "faces" chemisorption is the rate controlling step followed by rapid decomposition of surface oxides. On the "edges" decomposition is the rate controlling step. This interpretation applied to the data yield $131 \mathrm{~kJ} / \mathrm{mol}$ for the activation energy of chemisorption and $150 \mathrm{~kJ} / \mathrm{mol}$ for decomposition $(31$ and $36 \mathrm{kcal} / \mathrm{mol}$, respectively).
\end{abstract}

Key words: Chemical anisotropy; chemisorption; oxidation; pyrolytic graphite.

\section{Introduction}

Pyrolytic graphite is of particular interest to those studying carbon because it may be obtained as sizable specimens with its anisotropic character intact. Thus, it may be studied by some of the simpler experimental techniques. Furthermore, those studies may be considered representative of the material as used in practice rather than of special experimental material. Because of the possible use in rocket nozzles and in leading edges of reentry space vehicles, the reaction with oxygen-containing gases has received study particularly questioning the chemical anisotropy.

In an article [1] ${ }^{1}$ about carbon and "borocarbon" deposited pyrolytically, Grisdale et al. tabulated relative reaction rates as $17: 1$ for the "ab-direction" compared to the "c-direction." (In this paper "cdirection" and "preferred c-direction" will be used interchangeably.) No explanation, experimental description, or other information was given. The same result was again tabulated in 1953 by Grisdale [2], also without explanation. Horton reported in 1961 [3] that this ratio was judged to be about 2.5 and to increase with increasing temperature. Levy and Wong [4] in

${ }^{1}$ Figures in brackets indicate the literature references at the end of this paper.
1964 reported a ratio of about 18 although in 1962 [5] Levy had reported that the oxidation of pyrolytic graphite proceeds preferentially in the c-direction. These appear to be the only studies of the rate ratio made on pyrolytic graphite although, as mentioned later, Lang and Magnier reported values for oxidation in damp air. The work of Strickland-Constable and colleagues $[6,7]$ concerned itself with the oxidation rate in the c-direction only and measured it directly without reference to the ratio. Horton's work was aimed at the c-direction rate also, but required at least a rough value for the ratio in order to make a needed correction to the measurements. Both Horton and StricklandConstable found that the c-direction rate was slower than the rate for the more usual synthetic graphites. This fact, of course, while not demonstrating a ratio different from unity, is consistent with such a possibility. These results may be contrasted with those of Gulbransen, Andrew, and Brassart [8] who found that above $1000{ }^{\circ} \mathrm{C}$ there was no difference in rate between pyrolytic and spectrographic graphites. However, they studied pyrolytic graphite only in the temperature region where gaseous diffusion controlled the rate. More recently, Lang and Magnier [24] reported rate ratios from 2.7 to 4.6 at $620^{\circ} \mathrm{C}$ when pyrolytic graphite was oxidized in air containing 0.02 volume percent 
water vapor. A temperature coefficient determination was not reported. The rate of reaction in dry air was said to be much less.

Single crystals of graphite would also be expected to exhibit chemical anisotropy and have been studied by Hennig [9]. He stated at first that the rate ratio was 20 below $800{ }^{\circ} \mathrm{C}$. In a later paper [18] he said the ratio must be at least 100 although the temperature was not specified. Thomas and Jones [10] found the ratio to be 26 at $800^{\circ} \mathrm{C}$. Oxidizing with nitric oxide gas they found essentially the same ratio and no essential difference for 873 or $910^{\circ} \mathrm{C}$. Patel and Bahl [11] measured rates of reaction for single crystals of Ticonderoga graphite in molten oxidizers: sodium peroxide, potassium nitrate, and sodium hydroxide between 350 and $650{ }^{\circ} \mathrm{C}$. The ratio of about 6.7 did not vary significantly with temperature.

Although the authors all agree that the rate ratio is greater than unity, there is disagreement about the magnitude and the existance of a temperature coefficient. One can remark at the outset that only Levy and Wong [4] and Horton [3] studied the ratio itself directly on macroscopic pyrolytic graphite and with oxygen. Other studies of the ratio were on single crystals $[9,10,11]$, probably on carbon films [1] or used other oxidizers [10, 11] or moist air [24]. It is not surprising that divergent results are obtained under these circumstances. The purpose of this paper is to present newer data and to examine in more detail the author's earlier data [3].

\section{Experimental Details}

The material used for study was from the same source as that used in 1960 , i.e., it constituted part of preparation Run 34 by the General Electric Company Research Laboratory. Prepared at $2100^{\circ} \mathrm{C}$ it had a density of $2.15 \mathrm{~g} \mathrm{~cm}^{-3}$. The quite low impurity levels were tabulated in the early reference [3]. The crystalline and microstructural characteristics of the material were determined using a high angle Geiger counter diffractometer for powder or powder-like patterns and using a three-circle automatic-scanning single-crystal (Eulerian cradle) diffractometer to determine the degree of preferred orientation. From the 002, 004, and 006 diffraction lines $c_{0}$ was determined to be $6.8326 \AA$ with a standard error of $0.0015 \AA$; $a_{0}$, from 10,11 , and 20 bands was $2.4527 \AA$ with standard error $0.0010 \AA$. Heights of layer stack from broadening of the 002, 004, and 006 lines (using $L_{c}=0.94 \lambda / \mathrm{B} \cos \theta$ ) [13] were respectively 106,92 , and $55 \mathrm{~A}$. From broadening of the 10 and 11 bands (using $L_{a}=1.77 \lambda / \mathrm{B} \cos \theta$ ) [14] the mean crystalline diameter $=146 \AA$, which compares reasonably with the value $128 \AA$ obtained from the apparent change in $a_{0}$ (using [14] $L_{a}=0.14 \lambda / \Delta(\sin \theta)$ ). Using Ergun's [15] designation for disk diameter as an alternative for $L_{a}$, we obtained $D=183 \AA$. From these data the number of layers in a stack is about 29 , and Franklin's [16] probability of layer misorientation, $p=0.85$. The degree of preferred orientation, when described according to Guentert and Cvikevich [17] as $I=I_{0}\left|\cos ^{m} \delta\right|$, is given by $m=6$.
The determination of material characteristics by $\mathrm{x}$-ray techniques, was initially attempted with powder obtained from filings which passed through a number 400 sieve. This was unsatisfactory because an insufficient number of lines and bands appeared. Furthermore, the thin film required absorption corrections [21] which were large, probably inaccurate, and were tedious to calculate. A solid, flat speciman was resorted to which was about $5 \mathrm{~mm}$ thick. Because of the preferred orientation the specimen was run twice, once with the x-ray beam incident to the "faces" and once with it incident to the "edges." Corrections were made for absorption [21], preferred orientation [22], as well as instrumental and doublet broadening [23] although the latter were quite small. The alinement of the diffracting system was checked by obtaining a pattern for a specially prepared specimen of quartz powder. This powder, with particle size about $0.15 \mu \mathrm{m}$, was also used to determine the magnitude of instrumental broadening.

Disk-shaped specimens were cut, as before [3], with dimensions between 0.6 and $2 \mathrm{~cm}$ diameter and thicknesses from 0.5 to $5 \mathrm{~mm}$. Specimens were cleaved to provide fresh basal-plane surface. No pretreatment was applied before insertion in the apparatus. However, the normal course of a run required preheating in nitrogen during the time required to reach temperature. About $1 \mathrm{hr}$ was required in each case to establish a steady state before the nitrogen was replaced by a reacting gas.

The specimens were oxidized in a horizontal tube furnace controlled within $1 \mathrm{~K}$. The central hot zone was within $\pm 2 \mathrm{~K}$ for a 2 -in region. This region was carefully located and the specimens always placed in it. Temperature was measured by a platinum:platinum-10 percent rhodium thermocouple placed near the specimen and by using a type K3 potentiometer. The melting point of gold was checked satisfactorily in the same apparatus. Tank gases passed through flowmeters before going to the furnace. The gases entered the furnace through a ceramic plug about $4 \frac{1}{2} 2$ in long. The passageway through the plug made two helical revolutions before opening on the reaction chamber. The increased path length was to ensure that the gas attained the furnace temperature more closely and to avoid a cooling effect on the specimens.

In contrast to the work of 1960 , in which only one specimen was run at a time, two runs were made with two specimens, seventeen were with three specimens and three were made with four specimens. Specimens run simultaneously were chosen to have divergent ratios of "faces" and "edges." Dimensions and weights were measured before and after each run. Blank runs were deemed unnecessary because thermogravimetric monitoring in the earlier work [3] showed no mass change during the pre- and post-oxidizing periods. Dimensional changes on each specimen could be used, as in the 1960 experiments, to give rate ratios. The main interest, however, was to evaluate the rate constants, $k_{i}$, in the equation

$$
-\dot{m}=k_{1} A_{f}+k_{2} A \text {. }
$$


where $\dot{m}$ is the rate of mass change, $A_{f}$ and $A_{e}$ are the total face and edge areas for a specimen, respectively, and $k_{1}$ and $k_{2}$ are the face and edge rates. For the case of two specimens run simultaneously a direct solution is possible. For the other cases, least squares solutions for the $k$ 's also lead to estimates of random error. Dimensions were measured with a micrometer caliper, and masses were measured with a commercial microbalance. Mass changes were from about $790 \mu \mathrm{g}$ to $740 \mathrm{mg}$. Although specimens stayed cylindrical edges did not always remain uniformly perpendicular to the faces. However, average diameters could be used in such cases. The specimens were drilled with a small axial hole to accept a platinum wire for mounting. They were suspended on this as on a spit as shown diagramatically in figure 1. The assembly could be moved in and out of the the furnace easily as needed. Although some oxidation could be observed around the holes, the magnitude of the effect was negligible.

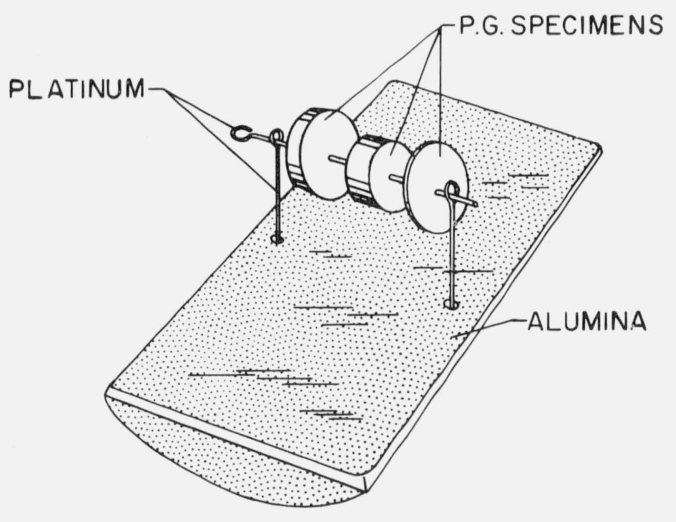

FIgURE 1. Specimens and support assembly.

The course of a run was as follows. Measured and weighed specimens were placed on the assembly and placed appropriately in the furnace. The gas entry plug was replaced and gas flows adjusted to be as planned. The oxidant was turned off and nitrogen allowed to continue while the furnace was heated and stabilized at the reaction temperature. At this point the oxidizing gas was turned on through a solenoid valve, simultaneously starting an electric clock. At the appropriate time the oxidizing gas was turned off, simultaneously stopping the clock. Throughout the run, readings of the gas flow and of the temperature were made. Total pressure for all runs was atmospheric. Air flows were from about 80 to $216 \mathrm{~cm}^{3} / \mathrm{min}$. Nitrogen flows, when used, were from about 80 to $300 \mathrm{~cm}^{3} / \mathrm{min}$. Because in the earlier work [3] flow rates changed by a factor of two had no significant effect, this factor was not investigated here. Partial pressures of oxygen were from about $1 / 10$ to $1 \mathrm{~atm}$. Times of exposure to the oxidizing gas were from 20 to 419 mins.

\section{Results}

The results of these experiments are shown in table 1 . The least squares solutions of the rate eq (1) were performed on a high speed electronic computer with the OMNITAB program [12] which provides also the standard errors of the rate constants. All other computations reported here were performed with this programming language also. Runs 3 and 4 have no entries for error because these were performed with only two specimens. Runs 20,21 , and 22 were those performed with four specimens; all others used three. It should be noted that runs 5 to 15 and run 17 were made with air. Run 16 was with pure oxygen. Runs 1 to 4 and 20 to 22 were made with air diluted with nitrogen. When examining the rate ratios these differences are probably unimportant. However, comparison among rate constants must, no doubt, take this into account. Three ratios are negative as a result of negative face rates. These together with runs 3,17 , and 19 were not considered further. Ratios for the latter three runs were demonstrably too scattered to be considered part of the group. The remaining 16 are shown graphically in figure 2 . The negative rates appear to be due to a combination of random error and small values.

As mentioned earlier sufficient dimensional data were taken to enable calculation of $\Delta D / \Delta H$ also, where $D$ is the diameter and $H$ the theikness of a specimen. Of the 67 specimens 41 gave apparently usable results and these are summarized in figure 3 . Of the remaining 26 , twenty-one specimens gave negative results, which

TABLE 1. Oxidation rates for pyrolytic graphite from mass changes

\begin{tabular}{|c|c|c|c|c|c|c|}
\hline Run & Temp. & $\begin{array}{c}\text { Face } \\
\text { rate, } k_{1}\end{array}$ & $\begin{array}{c}\text { Edge } \\
\text { rate, } k_{2}\end{array}$ & $\begin{array}{c}\text { Std. } \\
\text { error, } k_{1}\end{array}$ & $\begin{array}{c}\text { Std. } \\
\text { error, } k_{2}\end{array}$ & $\begin{array}{c}\text { Ratio, } \\
k_{2} / k_{1}\end{array}$ \\
\hline & kelvins & \multicolumn{4}{|c|}{$\mathrm{mg} \mathrm{cm}{ }^{-2} \mathrm{~min}^{-1}$} & \\
\hline 1 & 867 & $0.343-02$ & $0.113-01$ & $0.54-03$ & $0.15-02$ & 3.30 \\
\hline 2 & 891 & $.835-02$ & $.125-01$ & $.11-02$ & $.29-02$ & 1.50 \\
\hline 3 & 885 & $.794-03$ & $.128-01$ & $\ldots \ldots \ldots$ & .... & 16.05 \\
\hline 4 & 887 & $.910-02$ & $.208-01$ & ....... & ........ & 2.29 \\
\hline 5 & 888 & $.993-02$ & $.216-01$ & $.22-03$ & $.88-03$ & 2.17 \\
\hline 6 & 884 & $.198-01$ & $.266-01$ & $.33-03$ & $.13-02$ & 1.34 \\
\hline 7 & 887 & $.148-01$ & $.217-01$ & $.20-02$ & $.80-02$ & 1.47 \\
\hline 8 & 883 & $.999-02$ & $.154-01$ & $.83-03$ & $.33-02$ & 1.55 \\
\hline 9 & 882 & $.790-02$ & $.168-01$ & $.64-03$ & $.25-02$ & 2.12 \\
\hline 10 & 1214 & $.134+01$ & $.729+01$ & $.44-00$ & $.15+01$ & 5.46 \\
\hline 11 & 1044 & $-.377-01$ & $.108+01$ & $.88-01$ & $.34-00$ & -28.65 \\
\hline 12 & 1045 & $-.149-00$ & $.247+01$ & $.22-00$ & $.90-00$ & -16.56 \\
\hline 13 & 962 & $.720-02$ & $.115-01$ & $.24-02$ & $.67-02$ & 1.60 \\
\hline 14 & 961 & $.884-02$ & $.169-01$ & $.36-02$ & $.98-02$ & 1.90 \\
\hline 15 & 957 & $.110-01$ & $.206-01$ & $.23-02$ & $.65-02$ & 1.88 \\
\hline 16 & 1138 & $-.116+00$ & $.352+01$ & $.25-00$ & $.77-00$ & -30.24 \\
\hline 17 & 1136 & $.559-01$ & $.511+01$ & $.38-01$ & $.24-00$ & 91.38 \\
\hline 18 & 1136 & $.852-00$ & $.332+01$ & $.38-00$ & $.14+01$ & 3.89 \\
\hline 19 & 1137 & $.160+01$ & $.391-00$ & $.22-00$ & $.73-00$ & .24 \\
\hline 20 & 1047 & $.470-01$ & $.139-00$ & $.78-02$ & $.34-01$ & 2.96 \\
\hline 21 & 1047 & $.623-01$ & $.475-00$ & $.17-01$ & $.75-01$ & 7.63 \\
\hline 22 & 1045 & $.250-00$ & $.102+01$ & $.86-01$ & $.37-00$ & 4.07 \\
\hline
\end{tabular}

Computer-modified power-of-ten expression has been used for numbers. Thus, $0.343-02$ represents $0.343 \times 10^{-2}$. Precision is only roughly indicated by retained digits. The standard errors are better indicators. 


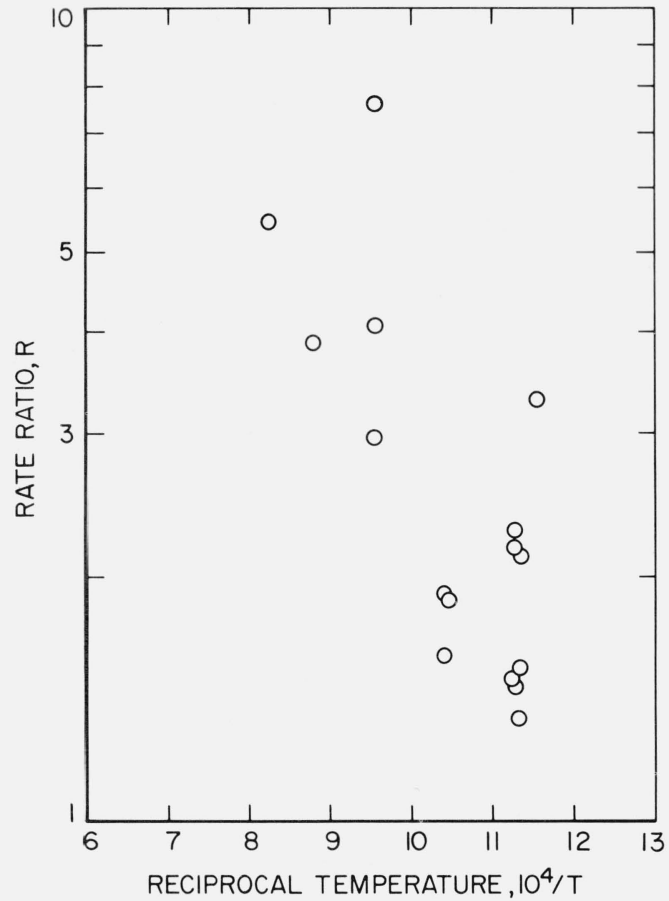

FigURE 2. Rate ratio versus reciprocal absolute temperatures for weight change data.

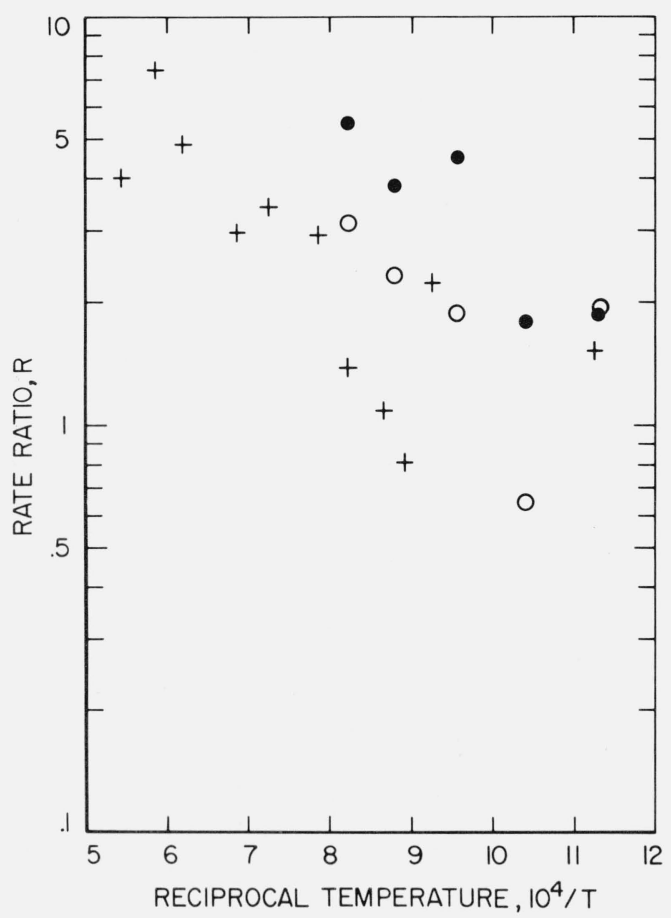

FIGURE 3. Rate ratio versus reciprocal absolute temperature: 1 - mass change data; 0 dimensional change data; + dimensional change data, 1960.

Note that within each set ratios have been averaged for closely neighboring temperatures in order to improve the clarity of the figure. arose, no doubt, from the smallness of the dimensional changes and the precision with which the dimensions could be measured. The precision of the latter measurements is probably most influenced by the uniformity of dimension at different points on a specimen. Five results appeared too far removed from the general trend to be considered.

Although the data obtained in 1960 were not specifically focused on the rate ratio the results are interesting to compare and are also represented in figure 3 . Here, there were 17 usable results out of 20 . Of the discarded three, two were negative and widely divergent. In what follows it will be useful to consider this group also. The data of table 1 are also shown in this figure 3 for comparison. Within each of the three sets some points are the average of results for closely neighboring temperatures for the sake of clarity.

\section{Discussion}

Although the precision of the data is poor, with adequate treatment some conclusions are possible. The main question is whether these data indicate that there is a temperature coefficient. The answer may be approached by fitting the data to a temperature dependent function and determining if the parameters of the function are significant. For this purpose it is convenient to use an equation of the Arrhenius type:

$$
R=\alpha e^{-\beta / T},
$$

where $R=k_{2} / k_{1}$. Although other forms may fit the data as well, this one corresponds to assuming that the rate ratio may be simply understood as the ratio of two rate constants, each of this form. Then $\alpha$ would represent the ratio of the preexponential factors and $\beta$ would represent the difference in the activation energies divided by the gas constant. For the three groups of data the results are given in table 2 . From the standard errors for the $\hat{\beta}$ 's and the numbers of degrees of freedom $(14,39$, and 15 , respectively), the probabilities that each of the nonzero slopes could have been obtained accidentally as a result of random error are about $0.0016,0.1$, and 0.015 , respectively. It appears to be beyond question that these data indicate the existence of a temperature coefficient for the oxidation rate ratio.

In order to understand the effect that is evident, it is

TABLE 2. Arrhenius parameters for rate ratio

\begin{tabular}{l|r|r|r|r|r}
\hline \hline \multicolumn{1}{c|}{ Data set } & $\widehat{\ln \alpha}$ & $s(\widehat{\ln \alpha})$ & $\hat{\beta}$ & $s(\hat{\beta})$ & $s(\widehat{\ln R})$ \\
\hline$\dot{m}$ & & & & & \\
$\Delta D / \Delta H$ & 4.69 & 0.95 & -3620 & 910 & 0.36 \\
$\Delta D / \Delta H, 1960$ & 2.37 & 1.04 & -1809 & 1060 & .70 \\
& 3.39 & .64 & -3180 & 820 & .46 \\
\hline
\end{tabular}

The expression $s(X)$ refers to the standard error of $X$. The symbol $\hat{x}$ refers to the estimate of $X$. The units of $\hat{\beta}$ and $s(\hat{\beta})$ are kelvins. All other data in this table are dimensionless. 
desirable to have a single set of values for the Arrhenius parameters. While attempting to do this, it became evident that although the three independent sets of data agree on the main conclusion there was some apparent discrepancy among them that suggested they should not be combined straight away. Further examination of the data showed that all three sets of data can be described by a single special equation. Expressed in the logarithmic form, the Arrhenius equation may be used with two intercepts and one slope:

$$
\ln R=\delta_{m} \alpha_{m}+\delta_{d} \alpha_{d}+\beta / T .
$$

In this equation $\delta_{m}$ is unity and $\delta_{d}$ is zero for mass change data, and conversely for dimensional data. This concept is essentially that of fitting a pair of parallel lines [19]. The values of the constants are given in table 3 . The parameters correspond to preexponential factors of 26.6 and 16.1, respectively, and to an activation energy difference of $-18900 \mathrm{~J}(-4500$ thermochemical calories) per gram atom of carbon. Accepting this interpretation of the data suggests that the simple relationships originally assumed (eqs 6, 7, 8 , and $k_{2} / k_{1}=\Delta D / \Delta H$ of ref. [3]) are not valid.

TABLE 3. Derived Arrhenius parameters

\begin{tabular}{ccc}
\hline Parameter & Value & $\begin{array}{c}\text { Standard } \\
\text { error }\end{array}$ \\
\hline$\hat{\alpha}_{m}$ & 3.28 & 0.54 \\
$\hat{\alpha}_{d}$ & 2.78 & .46 \\
$\hat{\beta}$ & $-2270 \mathrm{~K}$ & $500 \mathrm{~K}$ \\
\hline
\end{tabular}

The parameters correspond to preexponential factors of 26.6 and 16.1 , respectively, and to an activation energy difference of $-18900 \mathrm{~J}$ per gram atom of carbon $(-4500$ thermochemical calories per gram at om). The standard error of fitting $\ln \mathrm{R}$ to this set is 0.59

An explanation of the observed rate ratio is available in terms of the mechanism suggested by Nagle and Strickland-Constable [6]. For pressures of the order of 0.21 atm-oxygen their overall rate equation at a given temperature and oxygen pressure, $p$, reduces to:

$$
k=\frac{k_{A} p}{1+k_{z} p} .
$$

Only the "A-sites" are involved because at this pressure the fraction of surface sites which are the A-type is essentially unity at all the experimental temperatures. Equation (4) comes about as follows. The rate at which surface oxides are formed is given by $k_{A} \mathrm{p}(1-\theta)$, where $k_{A}$ is a rate constant for chemisorption and $\theta$ is the fraction of $A$-sites already covered by oxide. Sites are uncovered at a rate $k_{d} \theta$ where $k_{d}$ is the specific rate constant for decomposition and thus $k_{d} \theta$ is also the rate of product formation and of carbon loss. Assuming a steady state condition whereby the fraction of covered sites do not change, the two rates must be equal, leading to

$$
\theta=\frac{k_{A} p}{k_{d}+k_{A} p} \text {. }
$$

Multiplying by $k_{d}$ gives the rate which is seen to be eq (4) where $k_{z}=k_{A} / k_{d}$.

For the case at hand we assume the steady state condition for the "faces" but that decomposition is much more rapid than is adsorption. We can, therefore, assume that $k_{z} p \ll 1$, leading to $k_{1}=k_{A} p$. On the "edges" we assume that adsorption is so rapid that the rate controlling step is decomposition, and that $\theta$ is proportional to $p$. This leads to $k_{2}=k_{d} \kappa p$ where $\kappa$ is the proportionality constant, $\theta / p$. Correspondingly, $R=\kappa k_{d} / k_{A}=\kappa k_{z}^{-1}$. Although the constant, $\kappa$, was introduced to give the proportionality for the fraction of covered sites on the edges, it is easily seen that in reality one should talk about the specific number of sites in the preferred ab-direction and also in the c-direction. These are almost certainly quite different and in fact may account largely for the shift between adsorption and decomposition being rate controlling. The data of Lang and Magnier [24] suggest a value somewhere between 20 and 65 for the ratio of surface density of sites. The data of table 3 suggest about 20 for the ratio of preexponential factors. That we are probably dealing with the same kind of sites in both directions is reasonable in view of the fact that in pyrolytic graphite the "c-direction" is only a preferred direction. In as-deposited material there are still some crystallite edges available on the faces and vice versa. The constant, $\kappa$, must include a ratio of surface density of $A$-sites in the two directions.

Equating $R$ proportional to $k \bar{z}^{1}$ requires the activation energy difference to be $-17.2 \mathrm{~kJ}$ (4.1 kilocalories) per gram atom of carbon according to Nagle and Strickland-Constable. This is in remarkable agreement with the value reported here of -18.9 , and represents the difference in activation energy for decomposition and chemisorption with that for decomposition being the higher. The activation energy for chemisorption calculated from the data of Walker et al. [20], of 117 $\mathrm{Kj} / \mathrm{mol}(28 \mathrm{kcal} / \mathrm{mol})$ agrees reasonably with that for $k_{A}$ of Nagle and Strickland-Constable of $125 \mathrm{~kJ} / \mathrm{mol}$ (30 kcal $/ \mathrm{mol})$. The explanation developed above also requires that the face rate, $k_{1}$, be proportional to the rate of chemisorption and to the pressure. The corresponding values from table 1 yield $131 \mathrm{~kJ} / \mathrm{mol}$ with a standard error of $19 \mathrm{~kJ} / \mathrm{mol}(31.2$ and $4.6 \mathrm{kcal} / \mathrm{mol}$, respectively) for the activation energy of chemisorption, when negative values and the widely divergent run 3 are deleted from consideration. Runs 1 to 4 and 20 to 22 must be corrected by the ratio $(0.21 /$ pressure oxygen) to refer all to the pressure of oxygen in air. These ratios were 2 for runs 1 to 4 and respectively $4,401 / 101$, $4045 / 1045$ for the others, as determined from the flow rates of air and nitrogen. Using the data from this work only, the activation energy for decomposition of surface oxides is $150 \mathrm{~kJ} / \mathrm{mol}(36 \mathrm{kcal} / \mathrm{mol}$ ). The various activation energies are compared in table 4. 
TABLE 4. Activation energies

\begin{tabular}{c|cc|cc|cc}
\hline \hline & This research & \multicolumn{2}{|c|}{$\begin{array}{c}\text { Nagle \& Strick- } \\
\text { land-Constable }\end{array}$} & \multicolumn{2}{|c}{$\begin{array}{c}\text { Walker, } \\
\text { Austin \& Tietjen }\end{array}$} \\
\hline $\mathrm{E}_{\mathrm{z}}$ & 18.9 & $(4.5)$ & 17.2 & $(4.1)$ & $\ldots \ldots$ & $\ldots \ldots$ \\
$\mathrm{E}_{\mathrm{A}}$ & 131 & $(31)$ & 125 & $(30)$ & 117 & $(28)$ \\
$\mathrm{E}_{\mathrm{d}}$ & 150 & $(36)$ & 142 & $(34)$ & $\ldots \ldots$ & $\ldots \ldots$ \\
\hline
\end{tabular}

Units are: kilojoules per gram atom; (kilocalories per gram atom).

The author is grateful to P. Minard for her assistance in making the new rate measurements and to J. L. Winick for making the x-ray characterization measurements.

\section{References}

[1] Grisdale, R. O., Pfister, A. C., and van Roosbroeck, W., Bell Syst. Tech. J. 30, 271 (1951).

[2] Grisdale, R. O., J. Appl. Phys. 24, 1288-96 (1953).

[3] Horton, W. S., Proceedings of the Fifth Carbon Conference, Vol. 2, p. 233 (Pergamon Press, Oxford, 1963).

[4] Levy, M. and Wong, P., J. Electrochem. Soc. 111, 1088-91 (1964).

[5] Levy, M., I \& EC Product Research and Development 1, 19-23 (1962)

[6] Nagle, J. and Strickland-Constable, R. F., Proceedings of the Fifth Carbon Conference, Vol. 1, p. 154-64 (Pergamon Press, New York, 1961).

[7] Walls, J. R. and Strickland-Constable, R. F., Carbon 1, 333-38 (1964).
[8] Gulbransen, E. A., Andrew, K. F., and Brassart, F. A., J. Electrochem. Soc. 111, 626-27 (1964).

[9] Hennig, G. R., Proceedings of the Fifth Conference on Carbon, Vol. 1, 0. 143-46. (Pergamon Press, New York, 1963).

[10] Thomas, J. M. and Jones, K. M., J. Nucl. Mtls. 11, 236-39 (1964).

[11] Patel, A. R. and Bahl, O. P., J. Nucl. Mtls. 22, 226-27 (1967).

[12] Hilsenrath, J., Ziegler, G. G., Messina, C. G., Walsh, P. J., and Herbold, R. J., Nat. Bur. Stand. (U.S.), Handb. 101 , 284 pages (Mar. 1966), U.S. Govt. Printing Office, Washington, D.C. 20402

[13] Warren, B. E. and Bodenstein, P., Acta Cryst. 18, 282-86 (1965).

[14] Warren, B. E. and Bodenstein, P., Acta Cryst. 20, 602-05 (1966).

[15] Ergun, S., Carbon 6, 7-11 (1968)

[16] Franklin, R. E., Acta Cryst. 4, 253-61 (1951).

[17] Guentert, O. J. and Cvikevich, S. Proceedings of the Fifth Carbon Conference, Vol. 1, p. 473-84. (Pergamon Press, New York, 1962)

[18] Hennig, G. R., Zeit. fur Elektrochemie 66, 629-35 (1962).

[19] Dewdney, J. W. and Dewdney, S. H., Rev. Sci. Inst. 34, 436-37 (1963).

[20] Walker, P. L., Jr., Austin, L., and Tietjen, J., Chemistry and Physics of Carbon, Vol. 1, P. L. Walker, Jr., Ed., Chapter 6, (Marcel Dekker, New York, 1965).

[21] Keating, D. T. and Warren, B. E., Rev. Sci. Inst. 23, 519-22 (1952).

[22] Guentert, O. J. and Cvikevich S., Carbon 1, 309-13 (1964).

[23] Klug, H. P. and Alexander, L. E., X-ray Diffraction Techniques, Chap. 9, esp. pp. 494-521 (John Wiley \& Sons, New York, 1954).

[24] Lang, F. M. and Magnier, P., Chemistry and Physics of Carbon, Vol. 3, P. L. Walker, Jr., Ed., pp 121-209 (Marcel Dekker, New York, 1968).

(Paper 74A3-604) 